\title{
Neurobrucellosis: A Rare Cause for Spastic Paraparesis
}

\author{
Rishad Ahmed and Basanagouda S. Patil \\ Department of Medicine, Al Ameen Medical College; Karnataka, India
}

\begin{abstract}
A 41 year old man presented to the outpatient department with a three month history of difficulty in walking. He also had a history of positive sensory symptoms in the form of pins and needle sensation mostly below the waist. His symptoms had been progressive and there was no significant family history. He demonstrated a spastic gait and could only walk with assistance and support. DTR were hypertonic and sensory deficit was observed below twelfth dorsal vertebra. Sphincter abnormalities were present. Plantars were extensor bilaterally. Cerebral and spinal MRI with contrast was unremarkable. Brucella antigen titers were significantly high. CSF report was consistent with neurobrucellosis. After detailed analysis of his history, clinical picture and investigations the diagnosis of neurobrucellosis was made. Combined antimicrobial therapy was started, his neurologic condition gradually improved and he was able to walk without help after three months of treatment. Hence this case showed that neurobrucellosis may present as acquired progressive spastic paraparesis and it should always be borne in mind in patients with spastic paraparesis.
\end{abstract}

Key-Words: Spastic paraparesis, paraplegia, brucellosis, neurobrucella.

Brucellosis is a multisystem disease that may present with a broad spectrum of clinical manifestations and complications. Neurobrucellosis although rare is one of them. Patients with a Brucella infection occasionally manifest central nervous system involvement. Clinicians, especially serving in endemic areas or serving patients coming from endemic areas should consider neurobrucellosis in patients with unexplained neurological and psychiatric symptoms [1].

\section{Case Report}

A fourty-one year old man goat farmer by occupation presented with a three month history of difficulty in walking and pins and needle sensations below the waist. Symptoms were progressive and there was no significant family history of paraplegia. He had history of night sweats and decreased appetite. He visited some centre before where MRI brain and spine was done with normal study. Vitals were normal. Careful examination revealed palpable cervical groups of lymph nodes bilaterally. Respiratory and cardiovascular systems were normal. Hepatospleenomegaly and mild neck stiffness were present. Fundoscopy was normal. He had spastic gait and could walk with assistance and support. DTR were hypertonic, sensory deficit present below twelfth thoracic vertebra. Plantars were extensors bilaterally. ESR was raised to 32mm after first hour. Blood glucose, renal and liver function tests, thyroid profile, electrolytes were normal. Chest x-ray was normal. At this point of time the picture was a bit confusing and we decided to go for CSF fluid analysis and Brucella antigen titers. CSF showed pleocytosis with $189 \times 10^{6}$ cells/L, high protein levels and a

Received on 10 January 2009; revised 8 April 2009.

Address for correspondence: Dr. Rishad Ahmed. Department of Medicine, Al Ameen Medical College, Bijapur, Karnataka-586 108, India. Email:rishad14@gmail.com. Phone:91-8352-272502. Fax: 91-8352270184.

The Brazilian Journal of Infectious Diseases

2009;13(3):245. (C) 2009 by The Brazilian Journal of Infectious Diseases and Contexto Publishing. All rights reserved. low glucose level which indicated neurobrucellosis. Brucella antigen titers showed 1:320. Treatment was initiated with Doxycycline (200mg/d) and Rifampicin $(900 \mathrm{mg} / \mathrm{d})$ along with streptomycin (1gm/d IM) initially for fourteen days. Antidepressants were also added. Physiotherapy was advised. Condition gradually improved as he could walk without help after three months of treatment, when further treatment was stopped.

\section{Discussion}

Neurobrucellosis is an uncommon presentation of brucellosis whose features vary greatly and generally tend to be chronic. Laboratory procedures commonly used give negative results. Thus for brucellosis which is both treatable and curable, a high degree of suspicion is required, especially in endemic areas [2]. A study revealed positive history of raw milk ingestion, occupational contacts, handling infected materials, household contacts and veterinarians. Neurobrucellosis (18.86\%) manifested as polyradiculoneuropathy, myeloradiculopathy, meningo-encephalopathy and polyradiculo-myeloencephalopathy [3]. The changing risk pattern for this disease requires a high index of suspicion for early diagnosis and predictably favorable results to treatment [4]. Light Cycler based real time PCR assays in CSF samples are more rapid and sensitive than conventional tests and give rapid diagnosis of neurobrucellosis [5].

\section{References}

1. Yetkin et al. Evaluation of the clinical presentations in neurobrucellosis. Int J Infect Dis 2006 Nov;10(6):446-52.

2. Haji-Abdolbagi M. et al. Clinical and laboratory findings in neurobrucellosis: review of 31 cases. Arch Iran Med 2008 Jan;11(1):21-5.

3. Kochar D.K. et al. Hospital-based case series of 175 cases of serologically confirmed brucellosis in Bikaner. J Assoc Physicians India 2007 Apr; 55:271-5.

4. Reitman CA, Watters WC 3rd. Spinal brucellosis: case report in the United States. Spine 2002 May 1;27(9):E250-2.

5. Colmenero J.D. et al. Real time polymerase chain reaction: a new powerful tool for the diagnosis of neurobrucellosis. J Neurol Neurosurg Psychiatry 2005 Jul;76(7):1025-7. 